Journal of Mathematics and Statistics 4 (1): 37-40, 2008

ISSN 1549-3644

(C) 2008 Science Publications

\title{
Integral Dissipative Set-valued Maps
}

\author{
${ }^{1}$ Ogundiran, M. O. ${ }^{2}$ Ademola, T. A. \\ ${ }^{1}$ Department of Physical Sciences, Bells University of Technology, \\ PMB1015,Ota, Ogun State Nigeria \\ ${ }^{2}$ Department of Mathematics and Statistics, Bowen University, \\ PMB 284, Iwo, Osun State Nigeria
}

\begin{abstract}
Integral dissipativity arises over a period of time. This dissipativity for multivalued maps has some intrinsic properties together with their convexification. The space of Aumann integrable maps endowed with Hausdorff topology having this dissipativity condition is a complete metric space.
\end{abstract}

Keywords: Multifuctions, Aumann integral, Dissipativity, convex hull.

\section{INTRODUCTION}

There are different forms of dissipativity as considered in ${ }^{[1]},{ }^{[4]},{ }^{[5]},{ }^{[7]}$ and ${ }^{[8]}$. These dissipativity conditions have their relationships with monotonicity in the sense of Minty ${ }^{[6]}$, if a map $\mathrm{A}$ is maximal dissipative then -A is maximal monotone.However, there exists some multifunctions that are integral dissipative,but not necessarily dissipative. Such multifunctions exist on the attainable trajectories of neutral functional differential inclusions. Integrals of set-valued

maps (multivalued maps, multifunctions) were considered by Aumann in ${ }^{[2]}$, the properties of these integrals and their convex hull were established. In ${ }^{\text {[5] }}$ Kisielewicz considered some further properties of the space of Aumann integrable set-valued maps. He also considered integral dissipative set-valued maps. This dissipativity condition is applicable in the case where the dissipativity of a system is on the average over a (continuous) period of time.

In this work, we shall consider some properties of these integral dissipative set-valued maps, and show that their convex hull is also integral dissipative. We proved that the space of these maps is a complete metric space with respect to Hausdorff topology.

\section{Preliminaries}

\section{Set-Valued Maps}

Let $\mathrm{X}, \mathrm{Y}$ be sets, a map $F: X \rightarrow Y$ is said to be a setvalued map(multi-valued map, or multifunction) if for every $x \in X, F(x) \subset Y$. By a selection of a setvalued map $F$, we mean a single-valued map $f$, such that $f(x) \in F(x)$ for every $x \in X$. The basic properties of set-valued maps have been extensively considered in ${ }^{[1]}$ and ${ }^{[5]}$.

\section{Convex Hull}

Let $F: X \rightarrow Y$ be a set-valued map, the intersection of all the convex sets in $\mathrm{Y}$ containing $\mathrm{F}$ is called the convex hull of $\mathrm{F}$, it is denoted by $\operatorname{co}(\mathrm{F})$.

\section{The Space $(\operatorname{Comp}(X), h)$}

Let $\operatorname{Comp}(\mathrm{X})$ be the family of all non-empty compact subsets of a metric space $(X, \rho)$

Given $A, B \in \operatorname{Comp}(X)$, the Hausdorff distance, h, on $\operatorname{Comp}(\mathrm{X})$, is defined as:

$$
\begin{array}{r}
h(A, B)=\max \{\bar{h}(A, B), \bar{h}(B, A)\} \\
\text { Where, } \bar{h}(A, B)=\sup _{a \in A} \operatorname{dist.}(a, B) \\
\bar{h}(B, A)=\sup _{b \in B} \operatorname{dist.}(b, A)
\end{array}
$$

This distance function defines a metric on $\operatorname{Comp}(\mathrm{X})$ which induce a topology called the Hausdorff topology on $\operatorname{Comp}(\mathrm{X})$. Given a measurable set-valued map

$$
F: I \rightarrow \operatorname{Comp}\left(\Re^{n}\right)
$$

the set $\mathfrak{I}(F)$ of all L-integrable selections of $\mathrm{F}$ is said to be subtrajectory integrals of $\mathrm{F}$.

A measurable set-valued map $F: I \rightarrow \operatorname{Comp}\left(\Re^{n}\right)$, is said to be Aumann integrable on I if $\mathfrak{I}(F) \neq \phi$. 
The Aumann integral of $F$ over $I$ is defined by $\int_{I} F(t) d t=\left\{\int_{I} f(t) d t: f \in F, F \in \mathfrak{I}(F)\right\}$

The family of all Aumann integrable maps from I to $\mathfrak{R}^{n}$, shall be denoted by $\Lambda\left(I, \mathfrak{R}^{n}\right)$. A metric is defined on $\Lambda\left(I, \Re^{n}\right)$ as follows:

$d^{\prime}: \Lambda\left(I, \Re^{n}\right) \times \Lambda\left(I, \Re^{n}\right) \rightarrow \Re^{+}$,

$d^{\prime}(F, G)=\int_{I} h(F(t), G(t)) d t \quad, \quad$ for $\quad$ every

$F, G \in \Lambda\left(I, \Re^{n}\right)$. Let $X=\left(\Lambda\left(I, \Re^{n}\right), d^{\prime}\right)$ and $\operatorname{cl}(X)$, the space of all closed and bounded subsets of $\mathrm{X} . \mathrm{X}$ is a complete metric space, and hence $\left(\operatorname{cl}(X), \tau_{H}\right)$, where $\tau_{H}$ is a Hausdorff metric on $\operatorname{cl}(X)$ is complete (Theorem 1.1, [5]). In sequel by $c l(X)$, we mean the space. $\left(\operatorname{cl}(X), \tau_{H}\right)$.

\section{Integral dissipativity}

Let $K \subset X$, and $I=[\sigma, \sigma+a], a>0$. A setvalued map, $F: K \rightarrow c l(X)$, is said to be integral dissipative if for any $x \in K$, there exists $\delta_{0}>0$, such that for every $\delta \in\left(0, \delta_{0}\right) \quad, \quad h \in(0, \delta)$, $f, g \in F(x)$ and $\lambda>0$. The following inequality is satisfied :

$$
\begin{aligned}
& \int_{\sigma}^{\sigma+a-\delta}\left(\int_{s}^{s+h}|x(t)-x(s)| d t\right) d s \\
& \leq \int_{\sigma}^{\sigma+a-\delta}\left(\int_{s}^{s+h}|x(t)-x(s)-\lambda(f(t)-g(s))| d t\right) d s
\end{aligned}
$$

\section{RESULTS AND DISCUSSION}

The following results due to Aumann [2] shall be used to prove our main results.

\section{Lemma 1}

Let $I \subset \mathfrak{R}$,if $F: I \rightarrow \operatorname{comp}\left(\mathfrak{R}^{n}\right)$ is measurable and integrably bounded, then

$$
\int_{I} F(t) d t
$$

is a non-empty convex subset of $\mathfrak{R}^{n}$.

\section{Theorem (Aumann)}

Let $F: I \rightarrow \operatorname{comp}\left(\Re^{n}\right)$ be measurable and integrably bounded. Then

$$
\int_{I} F(t) d t=\int_{I} \operatorname{coF}(t) d t
$$

and both intervals are non-empty, convex compact subsets of $\mathfrak{R}^{n}$.

\section{Main Results}

We first showed that if a set-valued map $F$ is integral dissipative, so is its convex hull. We then showed that the space of all integral dissipative maps is complete.

\section{Theorem 1}

Let $K \subset X, \quad$ if $\quad F: K \rightarrow \operatorname{cl}(X) \quad$ is integral dissipative, then its convex hull $c o F$ is also integral dissipative.

\section{Proof}

From Aumann Theorem above, coF is Aumann integrable, since $\mathrm{F}$ is Aumann integrable Therefore, it suffces to show that coF satisfies the integral dissipativity condition. Since $\mathrm{F}$ is Aumann integrable, then for any $f, g \in F(x)$ and $\lambda>0, \delta_{0}>0$,

$$
\begin{aligned}
& \delta \in\left(0, \delta_{0}\right) ; \int_{\sigma}^{\sigma+a-\delta}\left(\int_{s}^{s+h}|x(t)-x(s)| d t\right) d s \\
& \leq \int_{\sigma}^{\sigma+a-\delta}\left(\int_{s}^{s+h}|x(t)-x(s)-\lambda(f(t)-g(s))| d t\right) d s
\end{aligned}
$$

But for any selection $f_{1} \in \operatorname{coF}(x)$, there exists selection $f_{2} \in F(x)$ such that $\int_{I} f_{1}(t) d t=\int_{I} f_{2}(t) d t$ , which follows from the fact that
$\int_{I} F(t) d t=\int_{I}^{I} c o F(t) d t$. Suppose $y \in \int_{I} \operatorname{co} F(t) d t$,
then $y=\int_{I} f(t) d t$, for some $f \in c o F$. By Caratheodory 's theorem, for each $t \in I$, the point $f(t) \in \operatorname{coF}(t)$, may be written as a convex combination of $\mathrm{n}+1$ points of $F(t)$, i.e. 


$$
\begin{aligned}
& f(t)=\sum_{i=0}^{n} \xi_{i}(t) f^{i}(t) \quad, \quad f^{i}(t) \in F(t), \\
& 0 \leq \xi(t) \leq 1, \sum_{i=0}^{n} \xi_{i}(t)=1
\end{aligned}
$$

Similarly, if $g(t) \in \operatorname{coF}(t), g(t)=\sum_{i=0}^{n} \omega_{i}(t) g^{i}(t)$, $g^{i}(t) \in F(t), 0 \leq \omega(t) \leq 1, \sum_{i=0}^{n} \omega_{i}(t)=1$.

Therefore for every $x \in K$, there exists $\delta_{0}>0$, such that for every $\delta \in\left(0, \delta_{0}\right), h \in(0, \delta) f^{i} g^{i} \in F(x)$ and $\lambda>0$ the inequality for each $\mathrm{i}$, holds since $\mathrm{F}$ is integral dissipative. Then for $f, g \in \operatorname{coF}(x)$

$$
\begin{aligned}
& \int_{\sigma}^{\sigma+a-\delta}\left(\int_{s}^{s+h}|x(t)-x(s)| d t\right) d s \leq \\
& \int_{\sigma}^{\sigma+a-\delta}\left(\int_{s}^{s+h} \mid x(t)-x(s)-\lambda\left[\sum_{i=0}^{n} \xi_{i}(t) f^{i}(t)-\omega_{i}(s) g^{i}(s)\right] d t\right) d s \\
& \leq \int_{\sigma}^{\sigma+a-\delta}\left(\int_{s}^{s+h}|x(t)-x(s)-\lambda(f(t)-g(s))| d t\right) d s
\end{aligned}
$$

for a suitable $\lambda>0$.

\section{Theorem 2}

Let $\left(F_{n}\right)$ be a Cauchy sequence of integral dissipative maps, which converges to $F$. Then $F$ is also integral dissipative.

\section{Proof}

We have to show that $\mathrm{F}$ is Aumann integrable and integral dissipative. Let $g_{n}$ be a selection of $F_{n}$, suppose $\left(g_{n}\right)$ is a Cauchy sequence. Then for every $\varepsilon>0$, there exists a positive integer $\mathrm{N}$ such that for every $m, n>N$, we have $d\left(g_{n}, g_{m}\right)<\varepsilon$

Take $\varepsilon=3^{-k}$ we can define an increasing sequence $n_{k}$ of positive integers such that $d\left(g_{n}, g_{n_{k}}\right)<3^{-k}$ for $m \geq n_{k}$ and $\mathrm{k}=1,2,3, \ldots$. In particular, we have $d\left(g_{n k+1}, g_{n_{k}}\right)<3^{-k}$, for $\mathrm{k}=1,2,3 \ldots$

We shall show that there is a measurable function $g: I \rightarrow \Re^{n}$ so that $h\left(g_{n_{k}}(t), g_{n}(t)\right) \rightarrow 0$ for a.e.

$$
\begin{aligned}
& \begin{array}{l}
t \in I \quad \text { as } \quad k \rightarrow \infty \text { Indeed, } \\
A_{k}=\left\{t \in I: h\left(g_{n_{k+1}}(t), g_{n_{k}}(t)\right) \geq 2^{-k}\right\} . \text { Then } \\
2^{-k} \mu\left(A_{k}\right) \leq \int_{A} h\left(g_{n_{k+1}}(t), g_{n_{k}}(t)\right) \\
\leq d\left(g_{n_{k+1}}, g_{n_{k}}\right) \\
<3^{-k}
\end{array} \\
& \text { Thus, } \mu\left(A_{k}\right)<\left(\frac{2}{3}\right)^{k} . \text { Putting } A=\bigcap_{m=1}^{\infty}\left(\bigcup_{k=m}^{\infty} A_{k}\right) \\
& \text { We have } \mu(A) \leq \mu\left(\bigcup_{k=m}^{\infty} A_{k}\right) \\
& \leq \sum_{k=m}^{\infty} \mu\left(A_{k}\right)<\sum_{k=m}^{\infty}\left(\frac{2}{3}\right)^{k} \\
& =3\left(\frac{2}{3}\right)^{m}
\end{aligned}
$$

let

for every $\mathrm{m}=1,2,3, .$. Then $\mu(A)=0$. Hence $\left\{g_{n_{k}}(t)\right\}$ is a cauchy sequence of measurable selections of some set-valued maps $\left\{G_{n_{k}}(t)\right\}$ in a

complete space $\operatorname{comp}\left(\Re^{n}\right)$ and by its completeness there exists $\tilde{G}(t) \in \operatorname{comp}\left(\Re^{n}\right), \tilde{g}(t) \in \tilde{G}(t)$, such that $h\left(g_{n_{k}}(t), \tilde{g}(t)\right) \rightarrow 0$ as $k \rightarrow \infty$

Taking this $\tilde{g}(t)=g(t)$ we conclude that the cauchy sequence $\left(g_{n}(t)\right)$ converges to $\mathrm{g}$.

Moreover, by Fatou's Lemma,

$$
\begin{gathered}
\int_{I} \lim _{i \rightarrow \infty} g_{i}(t) d t \leq \lim _{i \rightarrow \infty} \int_{I} g_{i}(t) d t \\
=\liminf _{i \rightarrow \infty} d\left(g_{n_{k}}, g\right) \leq 3^{-k}
\end{gathered}
$$

since $\quad n_{i} \geq n_{k} \quad$ Then $\quad \lim _{k \rightarrow \infty} d\left(g_{n_{k}}, g\right)=0$

$d\left(g_{n}, g\right) \leq d\left(g_{n}, g_{n_{k}}\right)+d\left(g_{n_{k}}, g\right)$

as $n \rightarrow \infty, d\left(g_{n}, g\right) \rightarrow 0$.

Then the limit $\mathrm{F}$ of $F_{n}$ is the family of the limits G of the sequence $\left(G_{n}\right)$. From the above, this family is Aumann integrable. Also, 


$$
\begin{aligned}
& \text { for any } \quad f, g \in F(x), \quad \text { such that } \\
& \lim _{n \rightarrow \infty} f_{n}=f \lim _{n \rightarrow \infty} g_{n}=g, f_{n}, g_{n} \in F_{n} \\
& \text { By }{ }^{\text {Fatou's }} \lim _{I}\left(f_{n}-g_{n}\right) d t \leq \lim _{n \rightarrow \infty} \int_{I}\left(f_{n}-g_{n}\right) d t \\
& \int_{\sigma}^{\text {But }} \\
& \leq \int_{\sigma}^{\sigma+a-\delta}\left(\int_{s}^{s+h}|x(t)-x(s)| d t\right) d s \\
& \leq \int_{\sigma}^{\sigma+a-\delta}\left(\int_{s}^{s+h}\left|x(t)-x(s)-\lambda \lim _{n \rightarrow \infty}\left(f_{n}(t)-g_{n}(s)\right)\right| d t\right) d s \\
&
\end{aligned}
$$

for each $\mathrm{n}$. Hence $\mathrm{F}$ is integral dissipative.

\section{CONCLUSION}

The space of Aumann integrable maps is sequentially complete with respect to Hausdorff toplogy. A subspace of this space with the property of integral dissipativity is also complete. Furthermore, the convex hull of integral dissipative multivalued map is also integral dissipative. This can be used to establish a relaxation theorem for integral inclusions having such multifunctions.

\section{REFERENCES}

1. Aubin J. P. and Cellina A..,(1984). Differential inclusion (set- valued maps and viability theory).Springer-Verlag Berlin.

2. Aumann R. J., (1956). Integrals of set-valued functions. J. Math. Anal. and Appl. 12, 1-12.

3 Bridgland I.R., (1970). Trajectory integrals of setvauled functions. Pacific J. Math. 33 , 43-67.

4 Kato T., (1967) Nonlinear semigroups and evolution equations. J. Math. Soc. Japan 19 508-520.

5 Kisielewicz M., (1991). Differential inclusion and optimal control, Kluwer Academic Publishers .

6. Minty G.,(1962). Monotone (nonlinear) operators in a Hilbert space. Duke Math. J. 29, 341-348.

7 Motyl J. (1995); On the solution of stochastic differential inclusion. J. Math. Anal. Appl. 192, 117-132.

8 Yosida K., (1980). Functional analysis, Sixth edition Springer-Verlag Berlin Heidelberg 